\title{
SURFACE TEMPERATURE RECONSTRUCTION BASED ON THE THERMOCAPILLARY EFFECT
}

\author{
M. SELLIER ${ }^{\otimes 1}$ and S. PANDA ${ }^{2}$
}

(Received 15 October, 2010; revised 19 April, 2011)

\begin{abstract}
A thin liquid film subject to a temperature gradient is known to deform under the action of thermocapillary stresses which induce convective cells. The free surface deformation can be thought of as the signature of the imposed temperature gradient, and this study investigates the inverse problem of trying to reconstruct the temperature field from known free surface variations. The present work builds on the analysis of Tan et al. ["Steady thermocapillary flows of thin liquid layers I. Theory", Phys. Fluids A 2 (1990) 313-321, doi:10.1063/1.857781] which provides a long-wave evolution equation for the fluid film thickness variation on nonuniformly heated substrates and proposes a solution strategy for the planar flow version of this inverse problem. The present analysis reveals a particular case for which there exists an explicit, closed-form solution expressing the local substrate temperature in terms of the local film thickness and its spatial derivatives. With some simplifications, this analysis also shows that this solution applies to three-dimensional flows. The temperature reconstruction strategies are successfully tested against "artificial" experimental data (obtained by solving the direct problem for known temperature profiles) and actual experimental data.
\end{abstract}

2000 Mathematics subject classification: primary 76T10; secondary 65L09.

Keywords and phrases: thin film, lubrication approximation, finite volume method, inverse problem.

\section{Introduction}

When the free surface of a thin liquid layer is subjected to a temperature gradient, a surface tension gradient balanced by shear stresses at the interface arises, resulting in fluid motion. Typically, convective cells develop, resulting in a deformation of the free surface which may be steady or unsteady, depending on the magnitude of the temperature gradient. Such flows have received much attention because of their fascinating nature and their relevance in a range of industrial applications such as the

\footnotetext{
${ }^{1}$ Department of Mechanical Engineering, The University of Canterbury, Private Bag 4800, Christchurch 8140, New Zealand; e-mail: mathieu.sellier@ canterbury.ac.nz.

${ }^{2}$ Department of Mathematics, National Institute of Technology, Calicut NIT Post. 673601, Kerala, India; e-mail: satyanand@ nitc.ac.in.

(C) Australian Mathematical Society 2011, Serial-fee code 1446-1811/2011 \$16.00
} 
growth of crystals in semiconductor materials, the rupture of thin films in heat transfer devices and the texturing of surfaces in magnetic storage devices [10].

In this work, we explore another possible application of such flows which involves estimating the substrate temperature from the free surface variations. Indeed, when a set of imposed conditions induces observable consequences, one can always take the reverse stand and attempt to deduce the imposed conditions from the observed consequences. Such a principle is exploited in the context of temperature-sensitive paint, for which the luminescent response of the paint to temperature variations is measured to produce temperature maps [7]. The question this work addresses is how the imposed temperature field can be reconstructed from a known deformation of the free surface. In order to solve this inverse problem and for the results to be of practical use, the following are needed:

(1) An accurate and efficient way of measuring free surface variations. We mention here the needle contact technique as used by Burelbach et al. [1] and Koehler [6], which provides pointwise values of the film thickness. Alternatively, optical techniques are routinely used to generate maps of free surface deformations. Such techniques include the shadowgraph technique [6, 13] and interferometry [3, 8].

(2) A reliable model to predict the liquid film thickness for a given temperature profile. Such free surface flows are typically investigated in the framework of the lubrication approximation, and Davis [2] and co-workers were the first to derive a long-wave evolution equation for the interface shape of a liquid layer on a heated plate for the purpose of studying Marangoni instability. We adopt the approach of Tan et al. [14]. Their theoretical investigation explored the effects of van der Waals forces, surface tension, surface tension gradients and gravity on the fluid film deformation in steady flows. The long-wave equation that they derived contains all of the physical phenomena relevant to a practical scenario for the inverse problem of interest here. More comprehensive models have since been developed $[5,9,11]$, but the work of Tan et al. offers the additional advantage that it comes with a companion experimental paper by Burelbach et al. [1] which provides the necessary experimental data to test and validate the developed concepts.

(3) A reliable algorithm to reconstruct the temperature profile from a known film thickness profile. Classically, such inverse problems are solved by defining an objective function giving a measure of the mismatch between the actual and simulated data. Then a sensitivity analysis is performed to find a direction in the parameter space which decreases the objective function. A minimum is sought by systematically following a decreasing direction of the objective function. This work shows that the inverse problem is governed by an ordinary differential equation (ODE) which may be readily integrated in a particular case. The methodology parallels that described by Sellier [12] to reconstruct the substrate profile from a known free surface variation. 


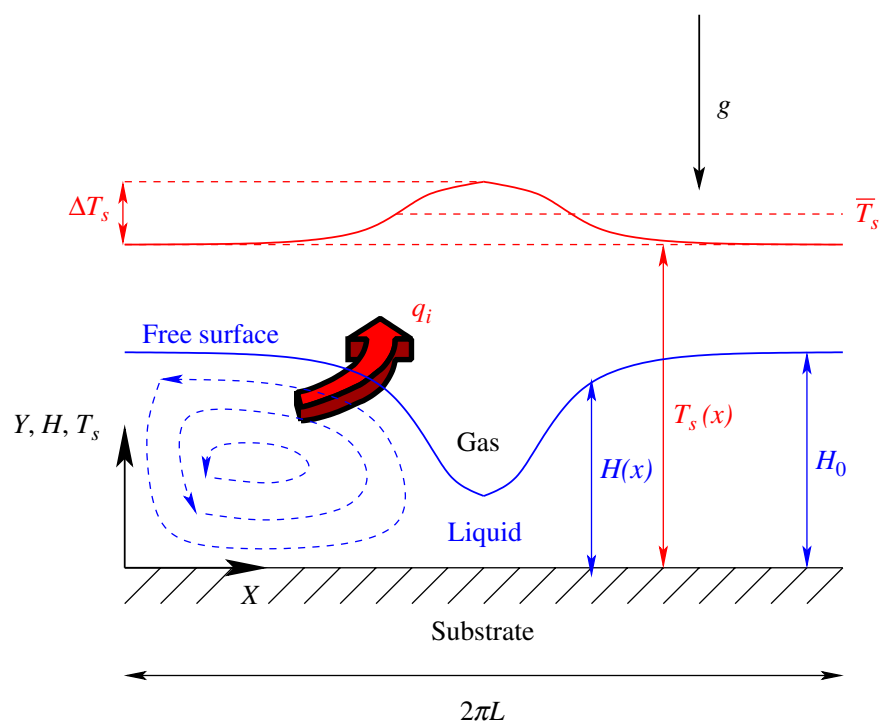

FIGURE 1. Schematic diagram of a thin liquid film flowing over a heated planar surface.

The paper is organised as follows. For completeness, the model derived by Tan et al. [14] for thermocapillary induced flows is summarized in Section 2. A description of the numerical method, with a validating case, is given in Section 3. The temperature reconstruction method is developed in Section 4. A report on a number of test cases is presented in Section 5, confirming the validity of the proposed methodology, and the paper closes with a discussion of the results and concluding remarks.

\section{The direct problem}

A typical flow scenario is illustrated in Figure 1. It shows a steady liquid film which lies over a horizontal planar heated surface. As the thin liquid layer is subjected to the temperature gradient imposed on the substrate, a surface tension gradient develops at the free surface, that is, regions of high temperature have a lower surface tension and regions of low temperature have a higher surface tension. The resulting spatial gradient in surface tension must be balanced by the shear stress at the interface, creating a flow within the liquid layer. This flow typically consists of convection cells, as illustrated.

The model developed by Tan et al. [14] used in the present work is described here for completeness. It is based on the lubrication approximation. The rigid plane lies at $Y=0$ and the liquid-gas interface lies at $Y=H(X)$, where $X$ and $Y$ are the in-flow and cross-flow coordinates, respectively. The rigid planar substrate is uniformly heated/cooled with its temperature $T_{S}(X)$ prescribed as a smooth $2 \pi L$ periodic function of $X$, and the ambient gas phase is air at constant temperature $T_{A}$. The mean substrate temperature is denoted by $\bar{T}_{S}$, and the difference between the maximum and minimum substrate temperature by $\Delta T_{s}$. 
The fluid is assumed incompressible and Newtonian with constant viscosity $\mu$, density $\rho$, specific heat $c_{p}$, thermal conductivity $K$ and thermal diffusivity $\kappa=K / \rho c_{p}$. The surface tension of the liquid-gas interface decreases linearly with the temperature $T$ according to

$$
\sigma=\sigma_{0}-\lambda\left(T-T_{0}\right)
$$

where $T_{0}$ is the reference temperature, $\sigma_{0}$ is the surface tension at $T=T_{0}$ and $\lambda$ is a positive constant specific to the fluid.

The heat flux $q_{i}$ at the interface is described by Newton's cooling law, namely

$$
q_{i}=\alpha\left(T-T_{A}\right),
$$

where $\alpha$ is the heat transfer coefficient that describes the rate of heat transport from the liquid to the ambient gas phase.

Following the analysis of Tan et al. [14], and scaling (i) the film thickness and pressure by $H_{0}$ (the mean thickness of the liquid layer over the interval $-\pi L \leq X \leq \pi L$ ) and $P_{0}=\rho\left(\mu / \rho H_{0}\right)^{2}$, respectively, (ii) the in-flow coordinate, cross-flow coordinate and time by $L, H_{0}$ and $T_{0}=L^{2} \rho / \mu$, respectively, and (iii) the temperatures of the liquid and substrate in terms of $\bar{T}_{S}$ and $\Delta T_{S}$, the lubrication equations for the dimensionless film thickness $h(x, t)$ and pressure $p(x, t)$ are given by

$$
\frac{\partial h}{\partial t}=\frac{\partial}{\partial x}\left(\frac{h^{3}}{3}\left(\frac{\partial p}{\partial x}+\frac{\mathrm{G}}{\mathrm{CPr}} \frac{\partial h}{\partial x}\right)+\frac{\mathrm{M} h^{2}}{2 \operatorname{Pr}} \frac{\partial}{\partial x}\left(\frac{T_{s}-\theta \mathrm{B} h}{1+\mathrm{B} h}\right)\right)
$$

and

$$
p=-\frac{a^{2}}{\operatorname{CPr}} \frac{\partial^{2} h}{\partial x^{2}} .
$$

Although the flows considered here are steady, the time dependency is retained in equation (2.1) to facilitate the numerical computation of the steady film profile.

The system is defined on the interval $-\pi<x<\pi$, and the periodic boundary condition

$$
h(-\pi, t)=h(\pi, t)
$$

is imposed on the model. The (dimensionless) similarity parameters are as follows:

- the Bond number $\mathrm{G}=\rho g H_{0}^{2} / \sigma_{0}$ expresses the ratio of body forces to surface tension forces;

- the Marangoni number $\mathrm{M}=\lambda H_{0} \Delta T_{s} / \mu \kappa$ characterizes the relationship between the temperature-dependent surface tension and viscous forces;

- the Prandtl number $\operatorname{Pr}=v / \kappa$ approximates the ratio of momentum diffusivity and thermal diffusivity;

- the Biot number $\mathrm{B}=H_{0} \alpha / K$ compares the relative magnitudes of resistances to internal conduction and surface convection;

- $\mathrm{C}=\mu \kappa / \sigma_{0} H_{0}$ is known as the Crispation number, $\theta=\left(\bar{T}_{S}-T_{A}\right) / \Delta T_{S}$ is the temperature ratio and $a=H_{0} / L \ll 1$ is the aspect ratio. 


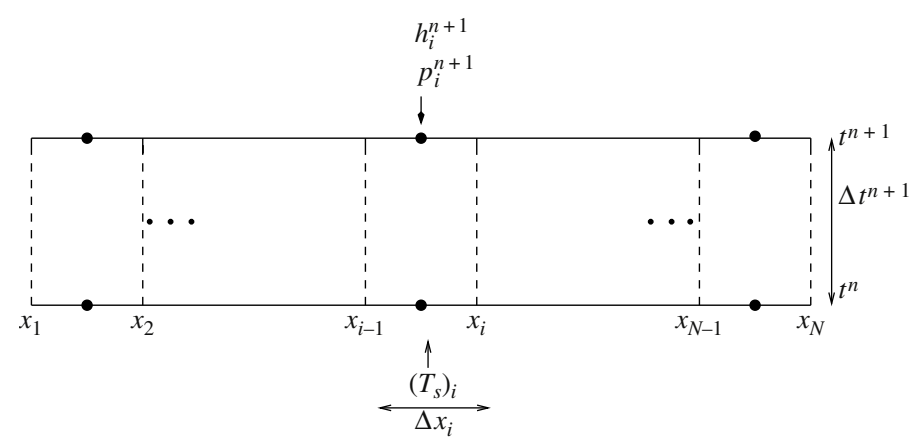

FIGURE 2. Typical grid used for finite volume discretizations. Black dots and dashed vertical lines represent the nodal points and cell faces, respectively.

\section{Solution procedure}

The coupled equations (2.1) and (2.2) for the film height and pressure together with the periodic boundary condition (2.3) are solved numerically. Many different numerical techniques have been proposed in the past to solve equations of this type; a good comparative study of the relative merits of the various discretization techniques is given by Ha et al. [4]. The most commonly employed discretization technique is that of finite differences or finite elements. In the present work we propose a new numerical scheme to solve the nonlinear equations (2.1) and (2.2), using the finite volume method [15] on a uniform grid with an implicit flux discretization.

We discretize the fluid domain using a uniform grid as shown in Figure 2. The flow variables $h$ and $p$ and the steady temperature $T_{s}$ are located at the cell centres. Let $N$ grid points be equidistributed in the interval $[-\pi, \pi]$, that is, $x_{i}=-\pi+(i-1) \Delta x_{i}$, $i=1, \ldots, N$, where $\Delta x_{i}=2 \pi /(N-1)$. The numerical solution is sought at the discrete time levels $t^{n}, n=0,1, \ldots$, with time step $\Delta t^{n+1}=t^{n+1}-t^{n}$.

To set up the discrete equations, the functions $h$ and $p$ are approximated over the cell $\left[x_{i-1}, x_{i}\right]$. Let $h_{i}^{n}$ and $p_{i}^{n}$ be the cell average of $h$ and $p$ in the cell $\left[x_{i-1}, x_{i}\right]$ at time $t^{n}$, that is,

$$
h_{i}^{n}:=\frac{1}{\Delta x_{i}} \int_{x_{i-1}}^{x_{i}} h\left(x, t^{n}\right) d x
$$

and

$$
p_{i}^{n}:=\frac{1}{\Delta x_{i}} \int_{x_{i-1}}^{x_{i}} p\left(x, t^{n}\right) d x .
$$

Given the solutions $h_{i}^{n}$ and $p_{i}^{n}$ at the time level $t^{n}$, the solutions at the next time level $t^{n+1}$ are obtained by integrating equations (2.1) and (2.2) over the space and time interval $\left[x_{i-1}, x_{i}\right] \times\left[t^{n}, t^{n+1}\right]$. This yields the discrete equations

$$
\left(h_{i}^{n+1}-h_{i}^{n}\right) \Delta x_{i}=\left(f_{i}^{n+1}-f_{i-1}^{n+1}\right) \Delta t^{n+1}
$$


and

$$
p_{i}^{n+1} \Delta x_{i} \Delta t^{n+1}=\left(g_{i}^{n+1}-g_{i-1}^{n+1}\right) \Delta t^{n+1}
$$

for nodes $i=1,2 \ldots, N-1$, where the auxiliary discrete function $f_{i}^{n+1}$ is given by

$$
f_{i}^{n+1}=\left(\frac{h^{3}}{3}\left(\frac{\partial p}{\partial x}+\frac{\mathrm{G}}{\mathrm{CPr}} \frac{\partial h}{\partial x}\right)+\frac{\mathrm{M} h^{2}}{2 \operatorname{Pr}} \frac{\partial}{\partial x}\left(\frac{T_{s}-\theta \mathrm{B} h}{1+\mathrm{B} h}\right)\right)\left(x_{i}, t^{n+1}\right),
$$

with

$$
\begin{aligned}
& \left(\frac{h^{3}}{3}\right)\left(x_{i}, t^{n+1}\right)=\frac{1}{3}\left(\frac{h_{i}^{n+1}+h_{i+1}^{n+1}}{2}\right)^{3}, \\
& \left(\frac{\partial p}{\partial x}\right)\left(x_{i}, t^{n+1}\right)=\frac{1}{\Delta x_{i}}\left(p_{i+1}^{n+1}-p_{i}^{n+1}\right)
\end{aligned}
$$

and

$$
\frac{\partial}{\partial x}\left(\frac{T_{s}-\theta \mathrm{B} h}{1+\mathrm{B} h}\right)\left(x_{i}, t^{n+1}\right)=\frac{1}{\Delta x_{i}}\left(\left(\frac{T_{s}-\theta \mathrm{B} h}{1+\mathrm{B} h}\right)_{i+1}^{n+1}-\left(\frac{T_{s}-\theta \mathrm{B} h}{1+\mathrm{B} h}\right)_{i}^{n+1}\right) .
$$

The implicit discretization of the function $g$ gives

$$
g_{i}^{n+1}:=\left(-\frac{a^{2}}{\operatorname{CPr}} \frac{\partial h}{\partial x}\right)\left(x_{i}, t^{n+1}\right)
$$

with

$$
\frac{\partial h}{\partial x}\left(x_{i}, t^{n+1}\right)=\frac{1}{\Delta x_{i}}\left(h_{i+1}^{n+1}-h_{i}^{n+1}\right) .
$$

The first and last nodes are the boundary nodes, and we therefore use the periodic boundary condition (2.3) for the approximation of equations (2.1) and (2.2). In brief, at the first node,

$$
\begin{aligned}
\left(\frac{h^{3}}{3}\right)\left(x_{1}, t^{n+1}\right) & =\frac{1}{3}\left(\frac{h_{1}^{n+1}+h_{N-1}^{n+1}}{2}\right)^{3}, \\
\left(\frac{\partial p}{\partial x}\right)\left(x_{1}, t^{n+1}\right) & =\frac{1}{\Delta x_{i}}\left(p_{1}^{n+1}-p_{N-1}^{n+1}\right), \\
\frac{\partial}{\partial x}\left(\frac{T_{s}-\theta \mathrm{B} h}{1+\mathrm{B} h}\right)\left(x_{1}, t^{n+1}\right) & =\frac{1}{\Delta x_{i}}\left(\left(\frac{T_{s}-\theta \mathrm{B} h}{1+\mathrm{B} h}\right)_{1}^{n+1}-\left(\frac{T_{s}-\theta \mathrm{B} h}{1+\mathrm{B} h}\right)_{N-1}^{n+1}\right)
\end{aligned}
$$

and

$$
\frac{\partial h}{\partial x}\left(x_{1}, t^{n+1}\right)=\frac{1}{\Delta x_{i}}\left(h_{1}^{n+1}-h_{N-1}^{n+1}\right) .
$$

The discrete values at the last node are approximated similarly.

Equations (3.1) and (3.2) are solved implicitly using the MATLAB routine fsolve, which uses a nonlinear least-squares algorithm to solve a system of nonlinear equations. To demonstrate the successful implementation of the finite volume algorithm, the numerical results are compared to those obtained by Tan et al. [14] 




FiguRE 3. Free surface profile for the flow over a planar heated surface, demonstrating the progression of the transient solution towards the steady-state solution. The parameters are $S^{\star}=a^{2} / \mathrm{G}=0.06861$, $R=3 \mathrm{MC} / 2 \mathrm{G}=1.255, \mathrm{Pr}=1$ and $\mathrm{B}=0$.

for the cosine temperature profile

$$
T_{s}(x)=\frac{\cos (x)}{2}
$$

with a combination of $a, \mathrm{G}, \mathrm{M}$ and $\mathrm{C}$ such that $a^{2} / \mathrm{G}=0.06861,3 \mathrm{MC} / 2 \mathrm{G}=1.255$, $\operatorname{Pr}=1$ and $\mathrm{B}=0$, which correspond to the parameter choices used by Tan et al., who used the notation $S^{\star}=a^{2} / \mathrm{G}$ and $R=3 \mathrm{MC} / 2 \mathrm{G}$. The simulation was performed using 201 grid points and a time step of 0.1. The results are shown in Figure 3. After an initial transient, the free surface quickly converges towards the results of Tan et al., thereby validating the proposed numerical approach.

\section{The inverse problem}

The inverse problem under consideration consists of finding the temperature function $T_{s}(x)$ corresponding to a given film thickness distribution $h(x)$. For a given steady state, the governing equations (2.1) and (2.2) can be combined to obtain an equation in terms of the film thickness profile $h$ as follows:

$$
\frac{d}{d x}\left(\frac{h^{3}}{3}\left(-\frac{a^{2}}{\mathrm{C}} \frac{d^{3} h}{d x^{3}}+\frac{\mathrm{G}}{\mathrm{C}} \frac{d h}{d x}\right)+\frac{\mathrm{M} h^{2}}{2} \frac{d}{d x}\left(\frac{T_{s}-\theta \mathrm{B} h}{1+\mathrm{B} h}\right)\right)=0 .
$$

Integrating equation (4.1), we obtain

$$
\frac{h^{3}}{3}\left(-\frac{a^{2}}{\mathrm{C}} \frac{d^{3} h}{d x^{3}}+\frac{\mathrm{G}}{\mathrm{C}} \frac{d h}{d x}\right)+\frac{\mathrm{M} h^{2}}{2} \frac{d}{d x}\left(\frac{T_{s}-\theta \mathrm{B} h}{1+\mathrm{B} h}\right)=C_{1},
$$

where $C_{1}$ is a constant of integration which, according to Tan et al. [14], is equal to zero when $h$ is an even function. In fact, one can easily show that temperature distributions 
which result in zero volume flux at the domain boundary lead to $C_{1}=0$. Rearranging equation (4.2) gives

$$
\frac{d T_{s}}{d x}=\left(\theta+\frac{T_{s}-\theta \mathrm{B} h}{1+\mathrm{B} h}\right) \mathrm{B} \frac{d h}{d x}+(1+\mathrm{B} h)\left(\frac{2 C_{1}}{\mathrm{M} h^{2}}-\frac{2 h}{3 \mathrm{M}}\left(-\frac{a^{2}}{\mathrm{C}} \frac{d^{3} h}{d x^{3}}+\frac{\mathrm{G}}{\mathrm{C}} \frac{d h}{d x}\right)\right) .
$$

In order to solve the inverse problem of reconstructing the substrate temperature from the free surface variations, we distinguish two cases:

(1) If $C_{1}=0$, equation (4.2) simplifies to

$$
-\frac{a^{2}}{\mathrm{G}} h \frac{d^{3} h}{d x^{3}}+h \frac{d h}{d x}+\frac{3 \mathrm{MC}}{2 \mathrm{G}} \frac{d}{d x}\left(\frac{T_{s}-\theta \mathrm{B} h}{1+\mathrm{B} h}\right)=0,
$$

and we find that equation (4.4) is an exact differential by noting that

$$
h \frac{d^{3} h}{d x^{3}}=\frac{d}{d x}\left(h \frac{d^{2} h}{d x^{2}}-\frac{1}{2}\left(\frac{d h}{d x}\right)^{2}\right) \text { and } h \frac{d h}{d x}=\frac{d}{d x}\left(\frac{1}{2} h^{2}\right)
$$

and rearranging (4.4) as follows:

$$
\frac{d}{d x}\left(-\frac{a^{2}}{\mathrm{G}}\left(h \frac{d^{2} h}{d x^{2}}-\frac{1}{2}\left(\frac{d h}{d x}\right)^{2}\right)+\frac{1}{2} h^{2}+\frac{3 \mathrm{MC}}{2 \mathrm{G}}\left(\frac{T_{s}-\theta \mathrm{B} h}{1+\mathrm{B} h}\right)\right)=0 .
$$

Integrating (4.5) yields

$$
-\frac{a^{2}}{\mathrm{G}}\left(h \frac{d^{2} h}{d x^{2}}-\frac{1}{2}\left(\frac{d h}{d x}\right)^{2}\right)+\frac{1}{2} h^{2}+\frac{3 \mathrm{MC}}{2 \mathrm{G}}\left(\frac{T_{s}-\theta \mathrm{B} h}{1+\mathrm{B} h}\right)=C_{2} .
$$

The constant $C_{2}$ can be evaluated from knowledge of the free surface profile and temperature at a point. Finally, rearranging (4.6), the substrate temperature function can be reconstructed as

$$
T_{s}(x)=\left(C_{2} \frac{2 \mathrm{G}}{3 \mathrm{MC}}+\frac{2 a^{2}}{3 \mathrm{MC}}\left(h \frac{d^{2} h}{d x^{2}}-\frac{1}{2}\left(\frac{d h}{d x}\right)^{2}\right)-\frac{\mathrm{G}}{3 \mathrm{MC}} h^{2}\right)(1+\mathrm{B} h)+\theta \mathrm{B} h .
$$

A remarkable feature of this analysis is that whilst the direct problem involves the solution of a complicated third-order differential equation, the inverse problem, in contrast, admits a closed-form solution. The latter may be further simplified if the capillary effect and cooling or heating by the overlying gas are neglected. The first assumption is equivalent to setting the mean surface tension $\sigma_{0}$ to zero, which cancels out the multiplying factor of the second term on the right-hand side of equation (4.7). The second hypothesis is equivalent to setting $\alpha$ to zero or, equivalently, setting the Biot number to zero. Consequently, (4.7) reduces to

$$
T_{s}(x)=\frac{\mathrm{G}}{3 \mathrm{MC}}\left(2 C_{2}-h^{2}\right) .
$$

Note that this solution is independent of the mean surface tension $\sigma_{0}$ since its presence in the dimensionless group $\mathrm{G}$ is cancelled out by its presence in the dimensionless group $\mathrm{C}$. 

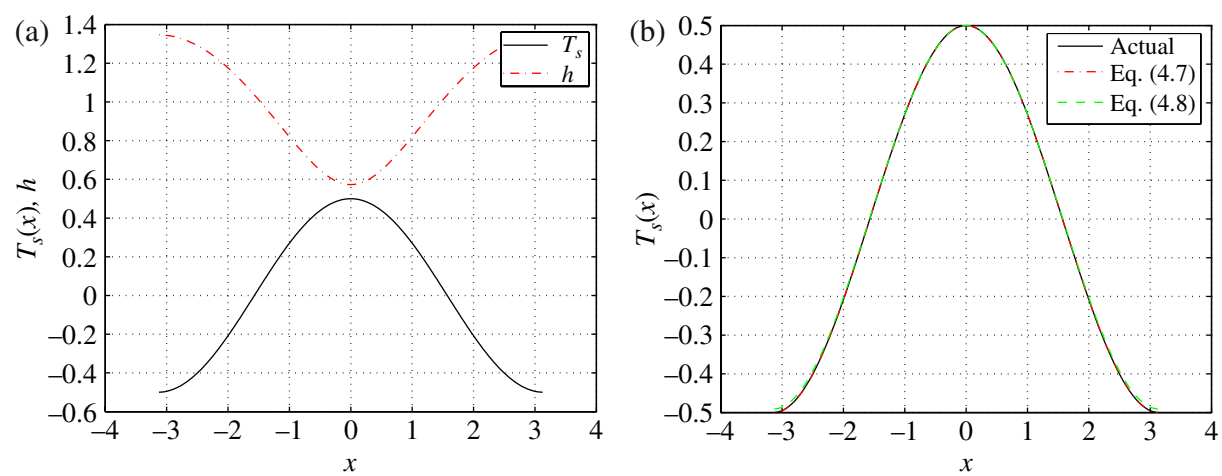

FIGURE 4. Results for the cosine temperature profile given by equation (3.3) and with $\mathrm{M}=5, \mathrm{G}=1, \mathrm{C}=$ $0.1, \mathrm{~B}=0.01, \theta=0.1$ and $a=0.02$ : (a) temperature and free surface profile; (b) actual and reconstructed temperature profiles according to equations (4.7) and (4.8).

(2) If $C_{1} \neq 0$, the inverse problem is explicitly governed by the first-order ODE (4.3), which involves the free surface function $h$ and its derivatives, together with the unknown constant $C_{1}$. In order to reconstruct the temperature profile, the following numerical procedure is used.

- In the first step, for any trial value of $C_{1}$, equation (4.3) is treated as an initial value problem and solved using the Runge-Kutta method over the domain $[-\pi, \pi]$.

- Next, the solution obtained with the trial value $C_{1}$ is used to integrate equation (4.1) and the result compared with the actual value of $C_{1}$. The Newton-Raphson method is used to update $C_{1}$, with tolerance $10^{-6}$.

This approach is referred to as the "ODE formulation" in the following section.

The numerical tests reported in the following section, based on the studies of Tan et al. [14] and Burelbach et al. [1], satisfy the condition $C_{1}=0$. When the algorithm proposed for the case $C_{1} \neq 0$ was tested, the reconstructed temperature profile was found to be indistinguishable from that obtained using the closed-form solution (4.7), and the constant $C_{1}$ was indeed found to be zero.

\section{Results and discussion}

In order to assess the validity of our approach, two tests were conducted. The temperature distribution is first reconstructed from an "artificial" film thickness profile obtained by first running the finite volume solver with a prescribed temperature profile. Secondly, the reconstruction formulas are tested by using the film thickness profile obtained experimentally by Burelbach et al. [1]. The results are summarized below.

5.1. Temperature reconstruction (artificial film thickness) For the first test case, the "experimental" film thickness distribution is obtained for the cosine temperature profile given by equation (3.3) with $\mathrm{M}=5, \mathrm{G}=1, \mathrm{C}=0.1, \mathrm{~B}=0.01, \theta=0.1$ and $a=0.02$. The steady-state solution, shown in Figure 4 , is obtained by solving the 

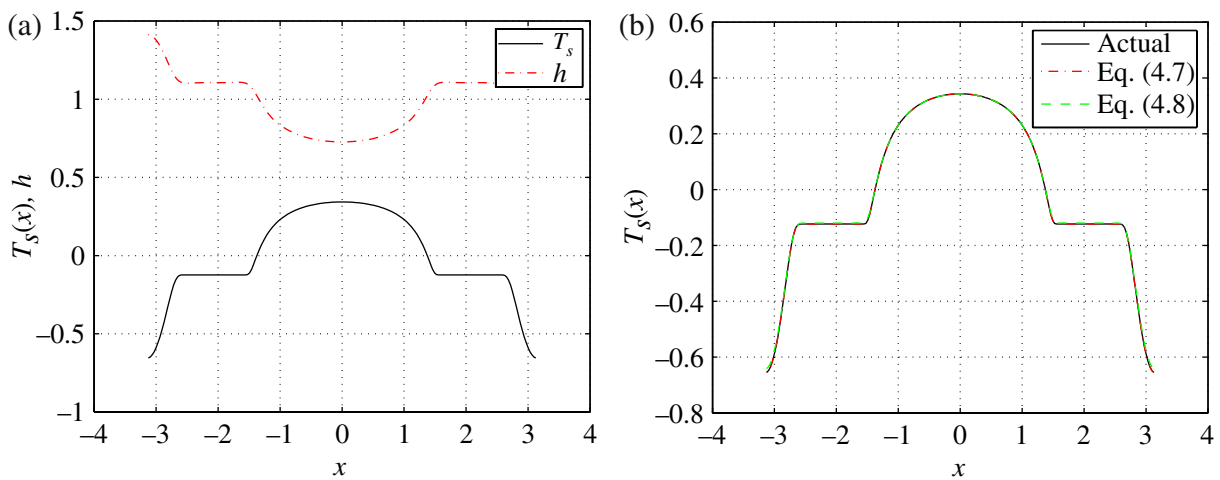

FIGURE 5. Results for the composite temperature profile given by equation (5.1) and with $\mathrm{M}=5$, $\mathrm{G}=1, \mathrm{C}=0.1, \mathrm{~B}=0.01, \theta=0.1$ and $a=0.02$ : (a) temperature and free surface profile; (b) actual and reconstructed temperature profiles according to equations (4.7) and (4.8).

system of partial differential equations (2.1) and (2.2) for 201 discrete points and a time step of 0.1. Because the film thickness is an even function here, the constant $C_{1}$ is equal to zero and the temperature reconstruction formulas (4.7) and (4.8) may be applied to test whether the temperature profile is successfully recovered. Standard centred difference formulas are used to calculate the first- and second-order derivatives in (4.7). The constant $C_{2}$ is evaluated from the knowledge of the film thickness profile and the temperature at a point. Figure 4(b) confirms that the reconstruction formulas are valid, since the reconstructed temperature profiles are indistinguishable from the prescribed one. For this particular case, the simple formula (4.8) provides an estimate of the temperature which is nearly as good as (4.7). This is a very convenient result as (4.8) does not require computation of derivatives, which is always an issue when dealing with true experimental data. An additional advantage is that the relative uncertainty of the reconstructed temperature profile varies linearly with the relative uncertainty of the measured film thickness.

The second example, also inspired by the work of Tan et al. [14], tests the reconstruction formulas for the thickness distribution resulting from the temperature profile

$$
T_{s}(x)= \begin{cases}0.7 \exp \left(\frac{4}{4 x^{2}-\pi^{2}}\right) & \text { for } 0 \leq|x|<\pi / 2 \\ -0.1235 & \text { for } \pi / 2 \leq|x| \leq 4 \pi / 5 \\ -0.1235-6.714 \exp \left(\frac{25}{25(|x|-\pi)^{2}-\pi^{2}}\right) & \text { for } 4 \pi / 5<|x| \leq \pi\end{cases}
$$

The temperature and free surface profiles, shown in Figure 5(a), are clearly well correlated, which explains the success of the reconstruction formula (Figure 5(b)).

5.2. Temperature reconstruction (experimental film thickness) Ultimately, the success of a reconstruction method needs to be assessed by considering real 

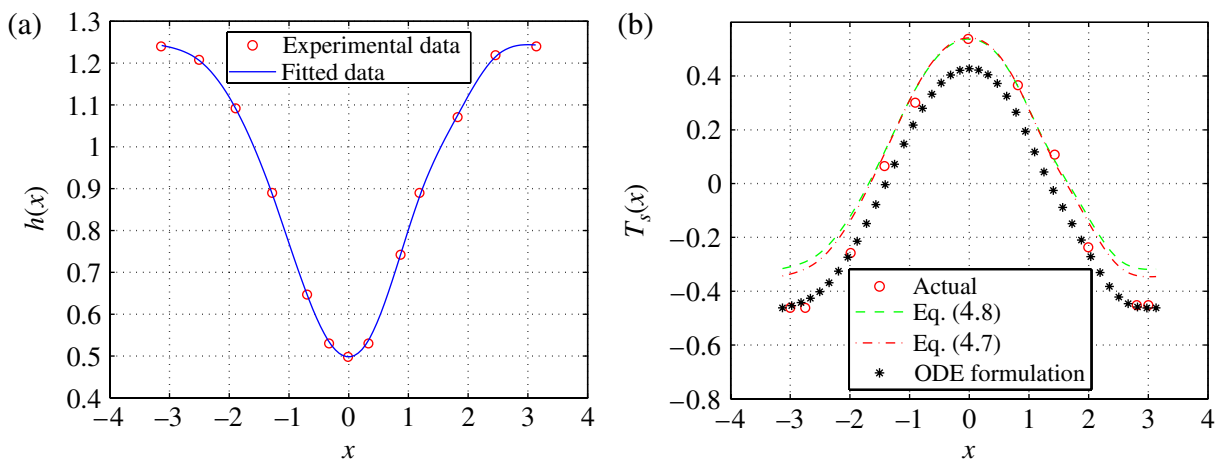

FIGURE 6. Temperature reconstruction for Run 3 of Burelbach et al. [1]: (a) measured film thickness with the corresponding fitted smoothing spline; (b) reconstructed temperature profile based on equations (4.7), (4.8), and using the ODE formulation with $\mathrm{G}=120, \mathrm{M}=148, \mathrm{C}=0.41, \mathrm{~B}=0.035, \theta=-0.32, a=0.03$.

experimental data. The work of Burelbach et al. [1] provides an ideal case study. These authors studied the deformation of the free surface of a thin layer of silicone oil resting on a horizontal substrate beneath which a strip layer was fastened. The mean thickness of the oil layer ranged from 0.125 to $1.684 \mathrm{~mm}$. The temperature distribution in the substrate was monitored by thermocouples and the interface shape by a mechanical impedance probe. The authors found that the lubrication approximation, as described by equations (2.1) and (2.2), could predict the film thickness to within $20 \%$. The aim of this section is therefore to establish whether the substrate temperature can be reconstructed to within the same accuracy, since clearly the inverse formulation cannot outperform the original model.

The first case considered corresponds to Run 3 of Burelbach et al. [1, Figure 7]. The data points were first read from the figure and a cubic smoothing spline was fitted through them using the MATLAB function csaps with a tolerance of $10^{-4}$. The resulting film thickness profile is shown in Figure 6(a). The reported values of the dimensionless parameters for this particular case are given in the figure caption. In order to reconstruct the temperature profile, the constant $C_{2}$ in equations (4.7) and (4.8) needs to be estimated. It is estimated at $x=0$ where information on the temperature and thickness profile is available simultaneously. Figure 6(b) shows the reconstructed temperature profiles according to (4.7), (4.8), and using the ODE formulation (4.3).

The quality of the reconstruction formula is assessed by considering the following performance indicator (PI), the ratio of the maximum difference between the reconstructed and actual temperature profiles to the actual temperature range:

$$
\mathrm{PI}=\frac{\max _{x \in[-\pi, \pi]}\left\|T_{s}^{\text {actual }}(x)-T_{s}^{\text {reconstructed }}(x)\right\|}{\Delta T_{s}} .
$$

Using the simple formula, which neglects capillary effects and the heating or cooling from the gas, a value of $17 \%$ is obtained for the PI. It decreases to $12.5 \%$ when the 

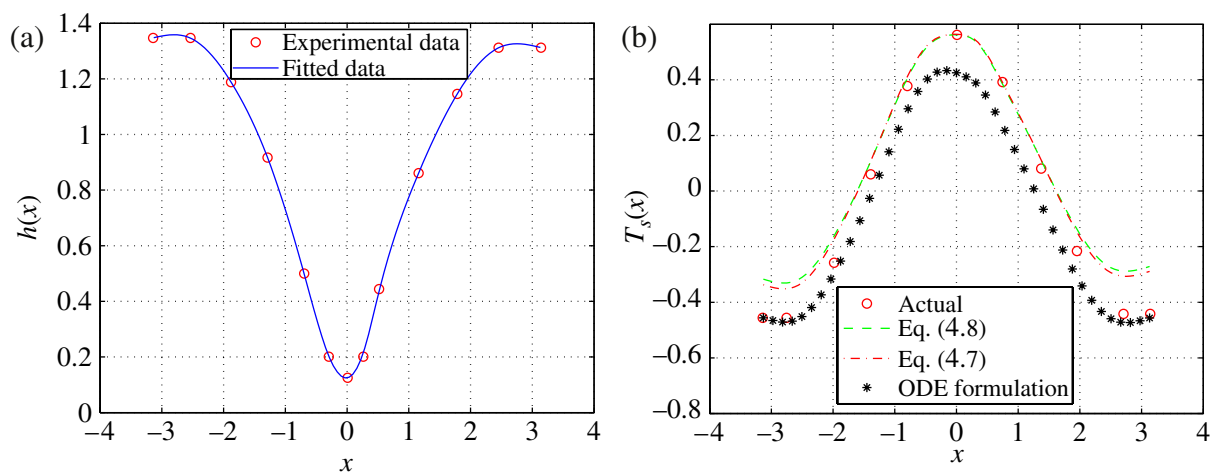

FiguRE 7. Temperature reconstruction for Run 4 of Burelbach et al. [1]: (a) measured film thickness with the corresponding fitted smoothing spline; (b) reconstructed temperature profile based on equations (4.7), (4.8), and using the ODE formulation with $\mathrm{G}=120, \mathrm{M}=200, \mathrm{C}=0.41, \mathrm{~B}=0.035, \theta=-0.11, a=0.03$.

full formula is used. This validates our analysis. As mentioned above, since the forward problem cannot estimate the film thickness to a better accuracy than $20 \%$, the inverse approach cannot be expected to outperform this result. Figure 6(b) also confirms the validity of the ODE formulation. The temperature profile is reconstructed assuming that $T_{s}(-\pi)$ is known and imposed as an initial condition for (4.3). The PI for the ODE formulation is $9 \%$, a slight improvement on the exact formulas. This improvement may be attributed to the fact that the ODE formulation does make the implicit assumption that the substrate temperature profile is periodic and the curve fitted through experimental readings is not enforced to be periodic.

The temperature reconstruction formulas are further tested on Run 4 from the experimental data of Burelbach et al. [1, Figure 8]. The measured film thickness profile and fitted smoothing spline are shown in Figure 7(a) for the dimensionless parameters indicated in the caption. The resulting reconstructed temperature profiles (Figure 7(b)) are in reasonable agreement with the actual, measured one. The PI for equations (4.7) and (4.8) is $14.3 \%$ and $17.5 \%$, respectively. It is $12 \%$ for the ODE formulation.

5.3. Extension to three-dimensional flows The previous section established that neglecting the capillary effects and the heating/cooling from the adjacent gas still provides a good estimate of the substrate temperature. A further benefit of this simple solution is that it applies equally well for three-dimensional flows. Indeed, it is straightforward to show that the simple solution (4.8) is also a solution of the corresponding system of equations for three-dimensional flows given by

$$
\begin{aligned}
\nabla \cdot \vec{q} & =0, \\
\vec{q} & =\frac{\mathrm{G}}{\mathrm{CPr}} \frac{h^{3}}{3} \nabla h+\frac{\mathrm{M}}{\operatorname{Pr}} \frac{h^{2}}{2} \nabla T_{s} .
\end{aligned}
$$

The first equation represents mass conservation in terms of the volumetric discharge $\vec{q}$ expressed in terms of gradients in hydrostatic pressure and Marangoni stresses in the 
second equation. The complete solution given by equation (4.7) does not, on the other hand, satisfy the corresponding system of equations for three-dimensional flows.

\section{Concluding remarks}

This paper demonstrates that it is possible to estimate the substrate temperature field from known free surface deformations induced by the thermocapillary effect. It is shown that the long-wave evolution equation for the interface shape derived by Tan et al. [14] can be rearranged to give an explicit ODE which governs the inverse problem. This ODE happens to be an exact differential for the "no-flux" particular case, and a closed-form expression for the local substrate temperature in terms of the local film thickness and its spatial derivatives is derived. This reconstruction formula is exact for planar flows, but an approximate formula is also proposed when the capillary effect and heating/cooling from the surrounding gas can be neglected. This approximate formula is shown to perform almost as well as the exact one for planar flows, the difference in the performance indicator (5.2) being of the order of $4 \%$ at most. The approximate formula has the advantage of being derivative-free, which is important when experimental data are involved, and, importantly, is shown to also apply for three-dimensional flows.

In all test cases, the accuracy of the reconstructed temperature was within $20 \%$, the estimated accuracy of the model of Tan et al. [14]. This obviously raises the question of when these reconstruction formulas can reliably be used. At least three, possibly important, phenomena are not accounted for in the present analysis: evaporation, inertia, and long-range molecular forces. The influence of these phenomena can be easily minimized if a fluid with low volatility is used, such as silicone oil, and if the thickness of the fluid layer is chosen not too small (typically greater than $0.1 \mu \mathrm{m}$ ), to avoid the interference of long-range molecular forces, nor too large, to remain in the inertia-free range. Tan et al. found that for silicone oil, inertia effects became nonnegligible for film thicknesses greater than $0.5 \mathrm{~mm}$. Despite this bounded applicability range and limited accuracy, the proposed idea could serve as an alternative strategy in the toolbox of heat transfer scientists and engineers and be used as a low-cost, low-tech temperature-sensitive coating.

\section{References}

[1] J. P. Burelbach, S. G. Bankoff and S. H. Davis, "Steady thermocapillary flows of thin liquid layers. II. Experiment”, Phys. Fluids A 2 (1990) 322-333, doi:10.1063/1.857782.

[2] S. H. Davis, "Thermocapillary instabilities", Annu. Rev. Fluid Mech. 19 (1987) 403-435, doi:10.1146/annurev.fluid.19.1.403.

[3] M. M. J. Decré and J. C. Baret, "Gravity-driven flows of viscous liquids over two-dimensional topographies”, J. Fluid. Mech. 487 (2003) 147-166, doi:10.1017/S0022112003004774.

[4] Y. Ha, Y.-J. Kim and T. G. Myers, "On the numerical solution of a driven thin film equation", J. Comput. Phys. 227 (2008) 7246-7263, doi:10.1016/j.jcp.2008.04.007.

[5] S. Kalliadasis, A. Kiyashko and E. A. Demsekhin, "Marangoni instability of a thin liquid film heated from below by a local heat source”, J. Fluid Mech. 475 (2003) 377-408, doi:10.1017/S0022112002003014. 
[6] T. P. Koehler, Experimental and numerical investigation of thermocapillary effects in thin liquid layers, Ph.D. Thesis, Georgia Institute of Technology, 2007.

[7] T. Liu, B. T. Campbell, S. P. Burns and J. P. Sullivan, "Temperature- and pressure-sensitive luminescent paints in aerodynamics", Appl. Mech. Rev. 50 (1997) 227-246, doi:10.1115/1.3101703.

[8] W. M. Nozhat, "Measurement of liquid-film thickness by laser interferometry", Appl. Optics 36 (1997) 7864-7869, doi:10.1364/AO.36.007864.

[9] C. Ruyer-Quil, B. Scheid, S. Kalliadasis, M. G. Velarde and R. Kh. Zeytounian, "Thermocapillary long waves in a liquid film flow. Part 1. Low-dimensional formulation", J. Fluid Mech. 538 (2005) 199-222, doi:10.1017/S0022112005005422.

[10] M. F. Schatz and G. P. Neitzel, "Experiments on thermocapillary instabilities", Annu. Rev. Fluid Mech. 33 (2001) 93-127, doi:10.1146/annurev.fluid.33.1.93.

[11] B. Scheid, A. Oron, P. Colinet, U. Thiele and J. C. Legros, "Nonlinear evolution of nonuniformly heated falling liquid films", Phys. Fluids 14 (2002) 4130-4151, doi:10.1063/1.1515270.

[12] M. Sellier, "Substrate design or reconstruction from free surface data for thin film flows", Phys. Fluids 20 (2008) 062106, (4 pages), doi:10.1063/1.2939404.

[13] G. S. Settles, Schlieren and shadowgraph techniques: visualizing phenomena in transparent media (Experimental fluid mechanics) (Springer, New York, 2001).

[14] M. J. Tan, S. G. Bankoff and S. H. Davis, "Steady thermocapillary flows of thin liquid layers. I. Theory", Phys. Fluids A 2 (1990) 313-321, doi:10.1063/1.857781.

[15] H. K. Versteeg and W. Malalasekera, An introduction to computational fluid dynamics: the finite volume method (Pearson Education, Harlow, UK, 1995). 